\title{
Testing universality of the Color Glass Condensate descriptions
}

\author{
M.S.Kugeratski ${ }^{1}$, V.P. Gonçalves ${ }^{2}$, and F.S. Navarra ${ }^{1}$ \\ ${ }^{1}$ Instituto de Física, Universidade de São Paulo, \\ C.P. 66318, 05315-970 São Paulo, SP, Brazil \\ ${ }^{2}$ High and Medium Energy Group (GAME), \\ Instituto de Física e Matemática, \\ Universidade Federal de Pelotas \\ Caixa Postal 354, CEP 96010-900, Pelotas, RS, Brazil
}

\begin{abstract}
Perturbative Quantum Chromodynamics (pQCD) predicts that the small- $x$ gluons in a hadron wavefunction should form a Color Glass Condensate (CGC), which has universal properties, which are the same for all hadrons or nuclei. Assuming this property, in this paper we cross relate the current CGC descriptions of the ep HERA data and $d A u$ RHIC data. In particular, we use the quark dipole scattering amplitude recently proposed by Kharzeev, Kovchegov and Tuchin (KKT) to explain the high $p_{T}$ particle suppression observed in $d A u$ collisions at RHIC in our calculations of the proton and longitudinal structure functions. We present a detailed comparison between this parameterization and those proposed to describe the ep HERA data. We find out that, due to its peculiar dependence on the energy and dipole separation, the KKT parameterization is able to describe the experimental $e p$ data only in a limited kinematical range of photon virtualities.
\end{abstract}

\section{INTRODUCTION}

In the past few years much theoretical effort has been devoted towards the understanding of the growth of the total scattering cross sections with energy. These studies are mainly motivated by the violation of the unitarity (or Froissart) bound by the solutions of the linear perturbative DGLAP [1] and BFKL [2] evolution equations. Since these evolution equations predict that the cross section rises obeying a power law of the energy, violating the Froissart bound [3], new dynamical effects associated with the unitarity corrections are expected to stop its further growth [4, [5]. This expectation can be easily understood: while for large momentum transfer $k_{\perp}$, the BFKL equation predicts that the mechanism $g \rightarrow g g$ populates the transverse space with a large number of small size gluons per unit of rapidity (the transverse size of a gluon with momentum $k_{\perp}$ is proportional to $1 / k_{\perp}$ ), for small $k_{\perp}$ the produced gluons overlap and fusion processes, $g g \rightarrow g$, are equally important. Considering the latter process, the rise of the gluon distribution below a typical scale is reduced, restoring the unitarity. That typical scale is energy dependent and is called saturation scale $Q_{s}$. The saturation momentum sets the critical transverse size for the unitarization of the cross sections. In other words, unitarity is restored by including non-linear corrections in the evolution equations [4, 5, 6, 7, 8, 9, 10, 11, 12, 13]. Such effects are small for $k_{\perp}^{2}>Q_{\mathrm{s}}^{2}$ and very strong for $k_{\perp}^{2}<Q_{\mathrm{s}}^{2}$, leading to the saturation of the scattering amplitude.

In the high energy limit, perturbative Quantum Chromodynamics (pQCD) predicts that the small- $x$ gluons in a hadron wavefunction should form a Color Glass Condensate (CGC), which is described by an infinite hierarchy of the coupled evolution equations for the correlators of Wilson lines [8, 9, 10, 11]. In the absence of correlations, the first equation in the Balitsky-JIMWLK hierarchy decouples and is then equivalent to the equation derived independently by Kovchegov within the dipole formalism [12]. A complete analytical solution of the Balitsky-Kovchegov (BK) equation is still lacking though there have been interesting recent developments in this direction (for recent reviews see, e.g. 14, 15, 16, 17]). A remarkable feature which emerges from the solution of this equation is that the dense, saturated system of partons to be formed in hadronic wave functions at high energy has universal properties, the same for all hadrons or nuclei. In particular, as the parton densities present in dAu collisions at RHIC are not too different from those measured in DIS at HERA, one expects CGC physics (and thus the presence of an energy dependent saturation scale $Q_{s}$ ) to affect particle production rates and cross sections. This allows us to cross relate these experiments in this respect and gain a clear understading of the CGC in high energy experiments. In order to illustrate this statement, in Fig. 1 we present the $A$ and $x$ dependence of the saturation scale, assuming the empirical parameterization $Q_{s}^{2}=A^{\frac{1}{3}} \times Q_{0}^{2}\left(\frac{x_{0}}{x}\right)^{\lambda}$, with the parameters $Q_{0}^{2}=1.0 \mathrm{GeV}^{2}, x_{0}=0.267 \times 10^{-4}$ and $\lambda=0.253$ as in Ref. 18]. We can observe that, while in the proton case we need very small values of $x$ to obtain large values of $Q_{s}^{2}$, in the nuclear case a similar value can be obtained for values of $x$ approximately two orders of magnitude greater. 


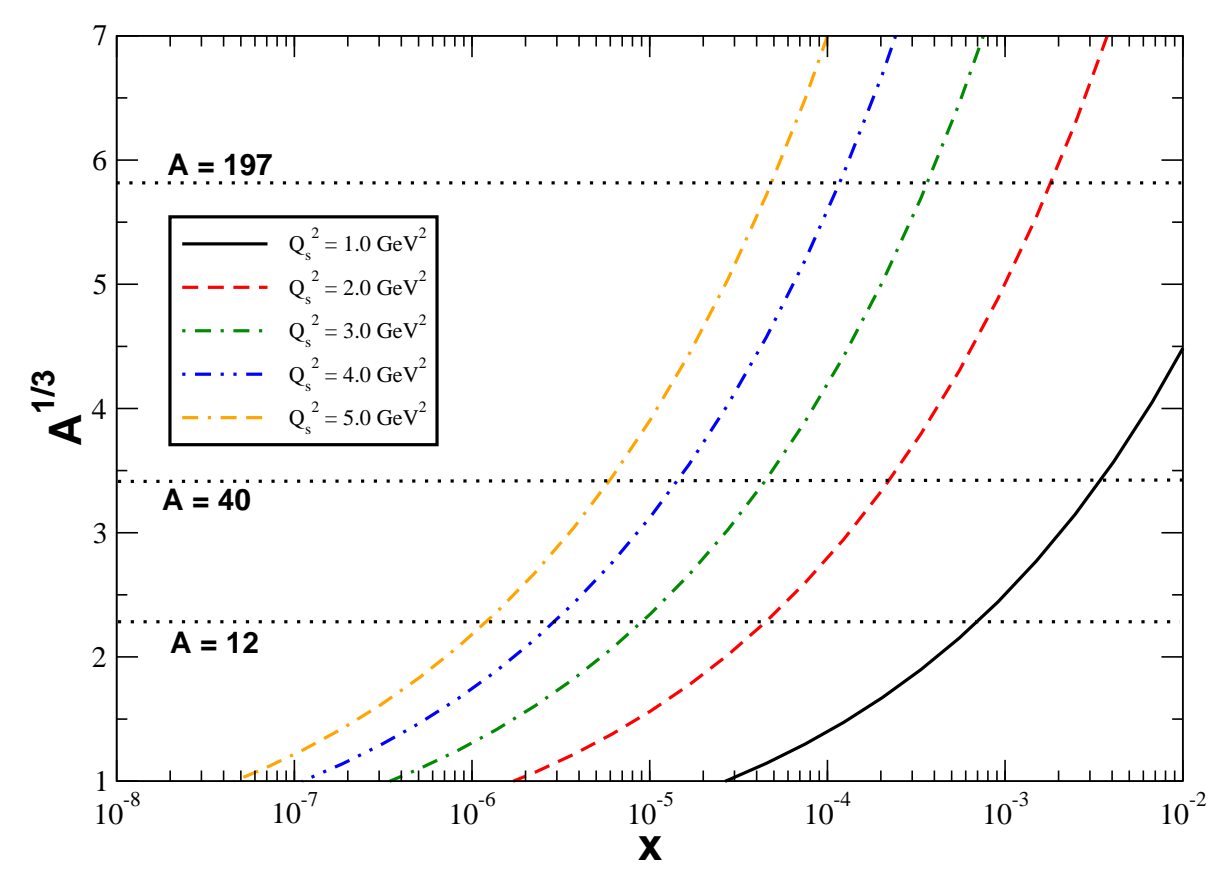

FIG. 1: Saturation scale for different values of $A$ and $x$.

In particular, the value of $Q_{s}^{2}=2 \mathrm{GeV}^{2}$, which is estimated from ep HERA data, can be obtained in $d A u$ collisions at RHIC in the forward rapidity region. A strong support for the universality of the CGC physics has been given recently in Ref. [19], which has noticed that the results for different collision systems in $\gamma^{*} p(A), d A$ and $A A$ can be related through the geometric scaling property, which is one of the main characteristics of the high density QCD approaches 20,21$]$.

The search of signatures for the parton saturation effects has been an active subject of research in the last years (for recent reviews see, e.g. 14, 17, 22]). In particular, it has been observed that the HERA data at small $x$ and low $Q^{2}$ can be successfully described with the help of saturation models [18, 23, 24, 25, 26]. Moreover, experimental results for the total cross section 27] and also for inclusive charm production 28] present the property of geometric scaling. On the other hand, the recently observed [29] suppression of high $p_{T}$ hadron yields at forward rapidities in dAu collisions at RHIC has the behavior anticipated on the basis of CGC ideas [30]. Although the data is qualitatively consistent with the predictions based on the CGC picture, only recently more quantitative analysis have been made 31, 32] (See also Refs. 19, 33 ). These approaches consider different basic assumptions in order to describe the experimental data. In particular, they consider distinct prescriptions for the dipole target cross section which is one of the basic elements of the CGC approaches. In Ref. 31] a generalization of the parameterization proposed by Iancu, Itakura and Munier (IIM) to describe the HERA data was used, obtaining a good agreement with the BRAHMS data on charged hadron production in the limited region of low transverse momenta and forward rapidity $(y=3.2)$. A comparison between this model and the RHIC data in the full kinematical range is not possible due to the behavior of the Fourier transform of the IIM dipole target cross section at intermediate transverse momenta 34 (For a recent detailed discussion of this subject see Ref. [35]). In [32], Kharzeev, Kovchegov and Tuchin (KKT) introduced a new parameterization with the free parameters fitted to RHIC data. In order to describe the hadron production in $d A u$ collisions at forward and mid-rapidities the authors has considered the contributions of gluon and valence quark production and convoluted it with the fragmentations functions and deuteron parton distributions. In particular, the gluon production cross section is given in terms of the gluon dipole scattering amplitude $\mathcal{N}_{G}(\boldsymbol{r}, x)$, while the valence quark production cross section is a function of the quark dipole scattering amplitude $\mathcal{N}_{Q}(\boldsymbol{r}, x)$. In principle both $\mathcal{N}_{G}(\boldsymbol{r}, x)$ and $\mathcal{N}_{Q}(\boldsymbol{r}, x)$ should be determined from the solution of the BK (or JIMWLK) evolution equation. However, as an analytical solution of this equation has not been accomplished so far, the authors from Ref. [32] have proposed a phenomenological parameterization for these two scattering amplitudes, inspired in the approximated analytical solutions of the BK equation for the saturation and color transparency regimes. It is important to emphasize that the pair separation and energy dependence proposed for the scattering amplitudes $\mathcal{N}_{G}(\boldsymbol{r}, x)$ and $\mathcal{N}_{Q}(\boldsymbol{r}, x)$ are identical, characterized by a $\left(\boldsymbol{r} Q_{s}^{2}\right)^{\gamma\left(Y, \boldsymbol{r}^{2}\right)}$ dependence, where the form of the anomalous dimension $\gamma\left(Y, \boldsymbol{r}^{2}\right)$ is constructed considering known analytical solutions to the BFKL equation. As our goal in this paper is to analyze the saturation physics in the 
deep inelastic scattering processes, which is directly associated to the quark dipole scattering amplitude, we will only consider the expression for $\mathcal{N}_{Q}(\boldsymbol{r}, x)$ proposed in Ref. [32]. The main uncertainty present in this procedure is associated with the normalization of the dipole cross section, which comes from the impact parameter dependence, and is not specified in [32]. In what follows we will consider the normalization as a free parameter to be fixed in a comparison with the experimental data and keep all other parameters fixed as in Ref. [32].

Based on the universality of the hadronic wave function, we might expect that the KKT parameterization would also describe the HERA data on proton structure functions. The main goal of this paper is to check this expectation. We will compare the predictions made with the KKT cross section and HERA ep data in the kinematical region where the saturation effects should be present ( small $x$ and low $Q^{2}$ ). Moreover, we analyze in detail the quark dipole scattering amplitude proposed in Ref. [32] and compare with those previously proposed to describe HERA data (for a related discussion see Ref. [36]). We observe that there are large differences in the energy and pair dipole size dependences of these models. We will arrive at the conclusion that the experimental data on proton structure function are described using the KKT prescription for the dipole cross section only in a limited kinematical range of photon virtualities. This result can be interpreted as an indicative that pre-asymptotic effects cannot be disregarded for the kinematical range of the RHIC and HERA colliders, which implies that the property of universality is still not manifested. It is important to emphasize that numerical studies of the BK equation show that its solution for intermediate rapidities presents a strong dependence in the choice for the initial condition [13, 37, 38]. Other important aspect that deserves more detailed analyzes is that the impact parameter dependence, which is disregarded in the phenomenological parameterizations, may have a significant effect on the behavior of the dipole scattering amplitudes.

A comment is in order here. A systematic comparison between the IIM and KKT dipole cross sections was started in Refs. [36] and [17], where possible ways to choose which one is the most appropriate were discussed. It was pointed out that one promising observable is the nuclear modification factor for photon production in deuteron-gold collisions at $y=3.8$. In [36] it was mentioned that the KKT parameterization had not been checked against DIS data on proton targets at HERA. In this paper we perform this check.

This paper is organized as follows. In the next section we shortly review the deep inelastic scattering in the color dipole picture, where the relation between the proton structure function $F_{2}\left(x, Q^{2}\right)$ and the dipole target cross section $\sigma_{\text {dip }}$ becomes explicit. Moreover, we review the parameterizations proposed in the literature for the quark dipole scattering amplitude, with particular emphasis on that proposed in Ref. 32. In Section III we present a detailed comparison between the distinct parameterizations for $\mathcal{N}(\boldsymbol{r}, x)$ discussed in the previous section. The asymptotic predictions for the color transparency and black disk regimes are compared as well as the energy and dipole size dependences. Furthermore, a comparison of predictions with the $F_{2}$ HERA data in the kinematical region of small values of $x$ and $Q^{2}$ is presented. As a by product, we also present a comparison with the HERA data on the longitudinal structure function. Finally, in Section IV we summarize our main results and conclusions.

\section{DEEP INELASTIC SCATTERING}

We start from the space-time picture of the eletron-proton/nuclei processes [39]. The deep inelastic scattering $e p(A) \rightarrow e+X$ is characterized by a large electron energy loss $\nu$ (in the target rest frame) and an invariant momentum transfer $q^{2} \equiv-Q^{2}$ between the incoming and outgoing electron such that $x=Q^{2} / 2 m_{N} \nu$ is fixed ( $m_{N}$ is the target mass). In terms of Fock states we then view the $e p(A)$ scattering as follows: the electron emits a photon $(|e>\rightarrow| e \gamma>)$ with $E_{\gamma}=\nu$ and $p_{t \gamma}^{2} \approx Q^{2}$. Afterwards the photon splits into a $q \bar{q}(|e \gamma>\rightarrow| e q \bar{q}>)$ and typically travels a distance $l_{c} \approx 1 / m_{N} x$, referred to as the coherence length, before interacting in the target. For small $x$, the photon is converted into a quark pair at a large distance before the scattering. Consequently, the space-time picture of the DIS in the target rest frame can be viewed as the decay of the virtual photon at high energy into a quark-antiquark pair (color dipole), which subsequently interacts with the target (for a review see, e.g., Ref. [40]). In the small $x$ region, the color dipole crosses the target with fixed transverse distance $\boldsymbol{r}$ between the quarks. The interaction $\gamma^{*} p(A)$ is further factorized and is given by [39],

$$
\sigma_{L, T}^{\gamma^{*} p(A)}\left(x, Q^{2}\right)=\sum_{f} \int d z d^{2} \boldsymbol{r}\left|\Psi_{L, T}^{(f)}\left(z, \boldsymbol{r}, Q^{2}\right)\right|^{2} \sigma_{d i p}^{p(A)}(x, \boldsymbol{r}),
$$

where $z$ is the longitudinal momentum fraction of the quark of flavor $f$. The photon wave functions $\Psi_{L, T}$ are determined from light cone perturbation theory and are given by

$$
\left|\Psi_{T}\left(z, \boldsymbol{r}, Q^{2}\right)\right|^{2}=\frac{6 \alpha_{\mathrm{em}}}{4 \pi^{2}} \sum_{f} e_{f}^{2}\left[z^{2}+(1-z)^{2}\right] \varepsilon^{2} K_{1}^{2}(\varepsilon r)+m_{f}^{2} K_{0}^{2}(\varepsilon r)
$$


and

$$
\left|\Psi_{L}\left(z, \boldsymbol{r}, Q^{2}\right)\right|^{2}=\frac{6 \alpha_{\mathrm{em}}}{\pi^{2}} \sum_{f} e_{f}^{2}\left\{Q^{2} z^{2}(1-z)^{2} K_{0}^{2}(\varepsilon r)\right\}
$$

The variable $\boldsymbol{r}$ defines the relative transverse separation of the pair (dipole) and $z(1-z)$ is the longitudinal momentum fraction of the quark (antiquark). The auxiliary variable $\varepsilon^{2}=z(1-z) Q^{2}+m_{f}^{2}$ depends on the quark mass, $m_{f}$. The $K_{0,1}$ are the McDonald functions and the summation is performed over the quark flavors.

The dipole hadron cross section $\sigma_{\text {dip }}$ contains all information about the target and the strong interaction physics. In the Color Glass Condensate (CGC) formalism 9, 10, 11], $\sigma_{\text {dip }}$ can be computed in the eikonal approximation and is given by:

$$
\sigma_{d i p}(x, \boldsymbol{r})=2 \int d^{2} \boldsymbol{b} \mathcal{N}(x, \boldsymbol{r}, \boldsymbol{b})
$$

where $\mathcal{N}$ is the quark dipole-target forward scattering amplitude for a given impact parameter $\boldsymbol{b}$ which encodes all the information about the hadronic scattering, and thus about the non-linear and quantum effects in the hadron wave function. The function $\mathcal{N}$ can be obtained by solving an appropriate evolution equation in the rapidity $Y \equiv \ln (1 / x)$. The main properties of $\mathcal{N}$ are: (a) for the interaction of a small dipole $\left(\boldsymbol{r} \ll 1 / Q_{\mathrm{s}}\right), \mathcal{N}(\boldsymbol{r}) \approx \boldsymbol{r}^{2}$, implying that this system is weakly interacting; (b) for a large dipole $\left(\boldsymbol{r} \gg 1 / Q_{\mathrm{s}}\right)$, the system is strongly absorbed and therefore $\mathcal{N}(\boldsymbol{r}) \approx 1$. This property is associated to the large density of saturated gluons in the hadron wave function. It is useful to assume that the impact parameter dependence of $\mathcal{N}$ can be factorized as $\mathcal{N}(x, \boldsymbol{r}, \boldsymbol{b})=\mathcal{N}(x, \boldsymbol{r}) S(\boldsymbol{b})$, so that $\sigma_{d i p}(x, \boldsymbol{r})=\sigma_{0} \mathcal{N}(x, \boldsymbol{r})$, with $\sigma_{0}$ being a free parameter related to the non-perturbative QCD physics.

Several models for the dipole cross section have been used in the literature in order to fit the HERA data. Here we will consider only the models proposed in Refs. [18, 23] which capture the main properties of the CGC physics. An equally good fit has been obtained in Ref. [38], where the $x$ dependence of the dipole cross section was derived from the numerical solution of the BK equation, including DGLAP corrections. In Ref. [23] Golec-Biernat and Wusthoff $(\mathrm{GBW})$ have proposed a phenomenological saturation model where $\mathcal{N}$ is given by

$$
\mathcal{N}(x, \boldsymbol{r})=\left[1-\exp \left(-\frac{\left(Q_{\mathrm{s}}(x) \boldsymbol{r}\right)^{2}}{4}\right)\right]
$$

with $Q_{s}^{2}=Q_{0}^{2} e^{\lambda \ln \left(x_{0} / x\right)}$. The parameters were obtained from a fit to the HERA data yielding $\sigma_{0}=23.03(29.12) \mathrm{mb}$, $\lambda=0.288(0.277)$ and $x_{0}=3.04 \cdot 10^{-4}\left(3.41 \cdot 10^{-4}\right)$ for a 3 -flavor (4-flavor) analysis [23]. An additional parameter is the effective light quark mass, $m_{f}=0.14 \mathrm{GeV}$, consistent with the pion mass. It should be noticed that the quark mass plays the role of a regulator for the photoproduction $\left(Q^{2}=0\right)$ cross section. The light quark mass is one of the non-perturbative inputs in the model. The charm quark mass is considered to be $m_{c}=1.5 \mathrm{GeV}$. A smooth transition to the photoproduction limit is obtained with a modification of the Bjorken variable as,

$$
\tilde{x}=x\left(1+\frac{4 m_{f}^{2}}{Q^{2}}\right)=\frac{Q^{2}+4 m_{f}^{2}}{W^{2}} .
$$

Observing Eq. (5) we notice that when $Q_{s}^{2}(x) \boldsymbol{r}^{2} \ll 1$, the model reduces to color transparency, whereas as one approaches the region $Q_{s}^{2}(x) \boldsymbol{r}^{2} \approx 1$, the exponential takes care of resumming many gluon exchanges, in a Glauberinspired way. Intuitively, this is what happens when the proton starts to look dark. Although the GBW parameterization gives a good description of the old HERA data, it has been ruled out by the new HERA data, with a much higher accuracy. This shortcoming is mainly related to the fact that this model fails to describe the Bjorken scaling violation and its functional form is only an approximation of the theoretical non-linear QCD approaches.

Another CGC inpired model has been proposed to described the HERA data in Ref. [18]. It is based on the understanding of the BFKL approach in the border of the saturation region [20]. In particular, the forward scattering amplitude has been calculated in both leading order (LO) and next-to-leading order (NLO) BFKL approaches in the geometric scaling region [41]. It reads

$$
\mathcal{N}(x, \boldsymbol{r})=\left[\boldsymbol{r}^{2} Q_{s}^{2}(x)\right]^{\gamma_{s}} \exp \left[-\frac{\ln ^{2}\left(\boldsymbol{r}^{2} Q_{s}^{2}\right)}{2 \beta \bar{\alpha}_{s} Y}\right],
$$

where the power $\gamma_{s}$ is the (BFKL) saddle point in the vicinity of the saturation line $Q^{2}=Q_{\mathrm{s}}^{2}(x)$. In this model the overall normalization of the dipole cross section is given by $\sigma_{0}=2 \pi R_{p}^{2}$, where $R_{p}$ is the proton radius. In addition, 
the anomalous dimension is defined as $\gamma=1-\gamma_{s}$. As usual in the BFKL formalism, $\bar{\alpha}_{s}=N_{c} \alpha_{s} / \pi$ and $\beta \simeq 28 \zeta(3)$. The quadratic diffusion factor in the exponential gives rise to the scaling violations, which are essential to describe the HERA data. As the forward scattering amplitude in Eq. (17) does not include an extrapolation from the geometric scaling region to the saturation region, the authors from Ref. [18] have constructed a parameterization for $\mathcal{N}(x, \boldsymbol{r})$ which smoothly interpolates between the limiting behaviors analytically under control: the solution of the BFKL equation for small dipole sizes, $\boldsymbol{r} \ll 1 / Q_{s}(x)$, and the Levin-Tuchin law [42] for larger ones, $\boldsymbol{r} \gg 1 / Q_{s}(x)$. A fit to the structure function $F_{2}\left(x, Q^{2}\right)$ was performed in the kinematical range of interest, showing that it is not very sensitive to the details of the interpolation (for a comprehensive phenomenological analysis of the HERA results using the numerical solution of the BK equation see Ref. 38]). The dipole-target forward scattering amplitude was parametrized as follows,

$$
\mathcal{N}(x, \boldsymbol{r})= \begin{cases}\mathcal{N}_{0}\left(\frac{\boldsymbol{r} Q_{s}}{2}\right)^{2\left(\gamma_{s}+\frac{\ln \left(2 / \boldsymbol{r}_{\left.Q_{s}\right)}\right.}{\kappa \lambda Y}\right)}, & \text { for } \boldsymbol{r} Q_{s}(x) \leq 2, \\ 1-\exp ^{-a \ln ^{2}\left(b \boldsymbol{r} Q_{s}\right)}, & \text { for } \boldsymbol{r} Q_{s}(x)>2,\end{cases}
$$

where the expression for $\boldsymbol{r} Q_{s}(x)>2$ (saturation region) has the correct functional form, as obtained either by solving the Balitsky-Kovchegov (BK) equation [9, 12], or from the theory of the Color Glass Condensate (CGC) 14]. Hereafter, we label the model above by IIM. The coefficients $a$ and $b$ are determined from the continuity conditions of the dipole cross section at $\boldsymbol{r} Q_{s}(x)=2$. The coefficients $\gamma_{s}=0.63$ and $\kappa=9.9$ are fixed from their LO BFKL values. In our further calculations we shall use the parameters $R_{p}=0.641 \mathrm{fm}, \lambda=0.253, x_{0}=0.267 \times 10^{-4}$ and $\mathcal{N}_{0}=0.7$, which give the best fit result. Recently, this model has also been used in phenomenological studies of vector meson production [43] and diffractive processes [44] at HERA as well as for the description of the longitudinal structure function [45].

On the other hand, Kharzeev, Kovchegov and Tuchin (KKT) have proposed a new parameterization for the dipole scattering amplitude in order to describe hadron production in dAu collisions [32]. As already discussed in the Introduction, in order describe the hadron production in $d A u$ collisions at forward and mid-rapidities these authors have proposed a phenomenological parameterization for the quark and gluon dipole scattering amplitudes, inspired in the approximated analytical solutions of the BK equation for the saturation and color transparency regimes. In this model the expression for the quark dipole-target forward scattering amplitude (hereafter $\mathcal{N}_{Q}=\mathcal{N}(\boldsymbol{r}, x)$ ) is given by 32]:

$$
\mathcal{N}(\boldsymbol{r}, x)=1-\exp \left[-\frac{1}{4}\left(\boldsymbol{r}^{2} \frac{C_{F}}{N_{c}} Q_{s}^{2}\right)^{\gamma\left(Y, \boldsymbol{r}^{2}\right)}\right] .
$$

where the anomalous dimension $\gamma\left(Y, \boldsymbol{r}^{2}\right)$ is

$$
\gamma\left(Y, \boldsymbol{r}^{2}\right)=\frac{1}{2}\left(1+\frac{\xi\left(Y, \boldsymbol{r}^{2}\right)}{\xi\left(Y, \boldsymbol{r}^{2}\right)+\sqrt{2 \xi\left(Y, \boldsymbol{r}^{2}\right)}+7 \zeta(3) c}\right),
$$

where $c$ is a free parameter and

$$
\xi\left(Y, \boldsymbol{r}^{2}\right)=\frac{\ln \left[1 /\left(\boldsymbol{r}^{2} Q_{s 0}^{2}\right)\right]}{(\lambda / 2)\left(Y-Y_{0}\right)}
$$

The authors assume that the saturation scale can be expressed by $Q_{s}^{2}(Y)=\Lambda^{2} A^{1 / 3}\left(\frac{1}{x}\right)^{\lambda}$. The form of the anomalous dimension is inspired by the analytical solutions to the BFKL equation [2]. Namely, in the limit $\boldsymbol{r} \rightarrow 0$ with $Y$ fixed we recover the anomalous dimension in the double logarithmic approximation $\gamma \approx 1-\sqrt{1 /(2 \xi)}$. In another limit of large $Y$ with $\boldsymbol{r}$ fixed, Eq. (10) reduces to the expression of the anomalous dimension near the saddle point in the leading logarithmic approximation $\gamma \approx \frac{1}{2}+\frac{\xi}{14 c \zeta(3)}$. Therefore Eq. (10) mimicks the onset of the geometric scaling region [18, 20]. In the calculations of Ref. [32] it is assumed that a characteristic value of $\boldsymbol{r}$ is $\boldsymbol{r} \approx 1 /\left(2 k_{T}\right)$ where $k_{T}$ is the transverse momentum of the valence quark and $\gamma$ was approximated by $\gamma\left(Y, \boldsymbol{r}^{2}\right) \approx \gamma\left(Y, 1 /\left(4 k_{T}^{2}\right)\right)$. As our goal is to apply this model to deep inelastic scattering, we explore two other possible approximations which are $\boldsymbol{r} \approx 1 / Q_{s}$ and $\boldsymbol{r} \approx 1 / Q$. In the above expressions the parameters $\Lambda=0.6 \mathrm{GeV}$ and $\lambda=0.3$ are fixed by DIS data 23. The initial saturation scale used in (11) is defined by $Q_{s 0}^{2}=Q_{s}^{2}\left(Y_{0}\right)$ with $Y_{0}$ being the lowest value of rapidity at which the low- $x$ quantum evolution effects are essential. When applied to describe RHIC data, the amplitude $\mathcal{N}(\boldsymbol{r}, x)$ must be convoluted with the quark distribution function in the hadron and with the fragmentation function of the quark. Moreover the gluon contribution must be added. These procedures introduce uncertainties in the predictions, which can only be estimated if the formalism is applied to other processes. 


\begin{tabular}{||c|c|c||}
\hline \hline & $\boldsymbol{r} \ll \boldsymbol{r}_{\text {sat }}$ & $\boldsymbol{r} \gg \boldsymbol{r}_{\text {sat }}$ \\
\hline \hline GBW & $\frac{\left(\boldsymbol{r} Q_{s}\right)^{2}}{4}$ & 1 \\
\hline IIM & $\mathcal{N}_{0}\left(\frac{\boldsymbol{r} Q_{s}}{2}\right)^{2}\left(\gamma_{s}+\frac{\ln \left(2 / \boldsymbol{r}_{Q}\right)}{\kappa \lambda Y}\right)$ & $1-e^{-a \ln ^{2}\left(b \boldsymbol{r} Q_{s}\right)}$ \\
\hline KKT & $\frac{1}{4}\left(\frac{C_{F}}{N_{c}}\left(\boldsymbol{r} Q_{s}\right)^{2}\right)^{\gamma\left(Y, \boldsymbol{r}^{2}\right)}$ & 1 \\
\hline \hline
\end{tabular}

TABLE I: Asymptotic limits of the quark dipole scattering amplitude in different models.

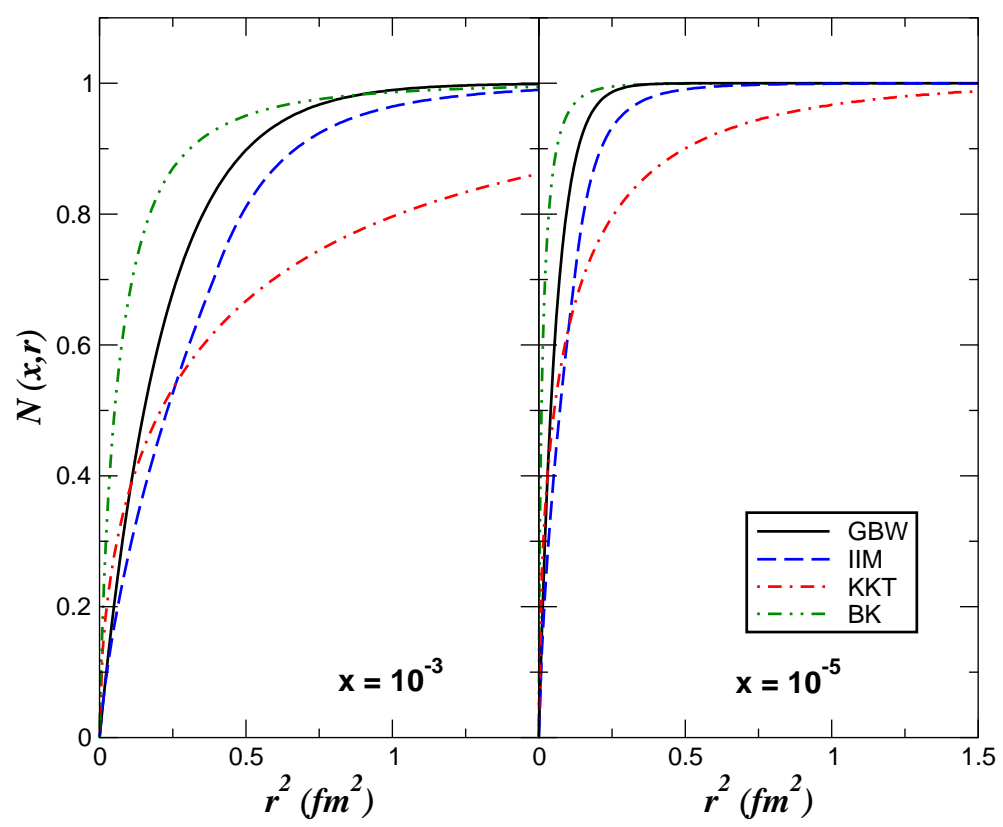

FIG. 2: Dependence of the quark dipole scattering amplitude in the squared pair separation $\boldsymbol{r}^{2}$ at different values of $x$.

A comment is in order here. The main goal of the IIM and KKT parameterizations is to mimick CGC physics in all kinematical regions. However, currently we have some theoretical control only on the asymptotic regimes of saturation and color transparency. Therefore we must assume some interpolation Ansatz in order to obtain a parameterization that may be used in practical calculations. Moreover, although both parameterizations have similar form, the KKT parameterization, in contrast to IIM one, includes the double logarithmic limit as well as the correct behavior in the saturation and geometric scaling regions. As is well known, this limit is important for large transverse momentum, allowing to connect the CGC physics with the DGLAP predictions. However, what is the correct linear limit (BFKL/DGLAP) in the kinematical regions of HERA and RHIC is still an open question.

\section{RESULTS AND DISCUSSION}

In this section we present a detailed study between the predictions of the distinct parameterizations for the quark dipole scattering amplitude and a comparison of its predictions with HERA data. We start presenting in Table 【 the asymptotic predictions for the linear regime $\boldsymbol{r} \ll \boldsymbol{r}_{\text {sat }}$ and saturation regimes $\boldsymbol{r} \gg \boldsymbol{r}_{\text {sat }}$, where $\boldsymbol{r}_{\text {sat }} \equiv 1 / Q_{s}$. As discussed in the Introduction, the critical line dividing dense and dilute regions is the saturation scale $Q_{s}$, with the property that the smaller the $x$, the denser the system gets and partons start to reinteract. The basic feature of the GBW, IIM and KKT models is that for a given $\boldsymbol{r}$, these models predict that the amplitudes tend to unity at small values of $x$ in contrast to the linear solution which predicts a exponential growth in this kinematical region. Moreover, all these models predict that the system saturates early, that is for large values of $x$ when the dipole size is larger. The three parameterizations present similar functional forms for the forward scattering amplitude in the two limits, with the IIM presenting a residual $\boldsymbol{r} Q_{s}$ dependence in the saturation regime, but also showing saturation for large values of $\boldsymbol{r} Q_{s}$. 


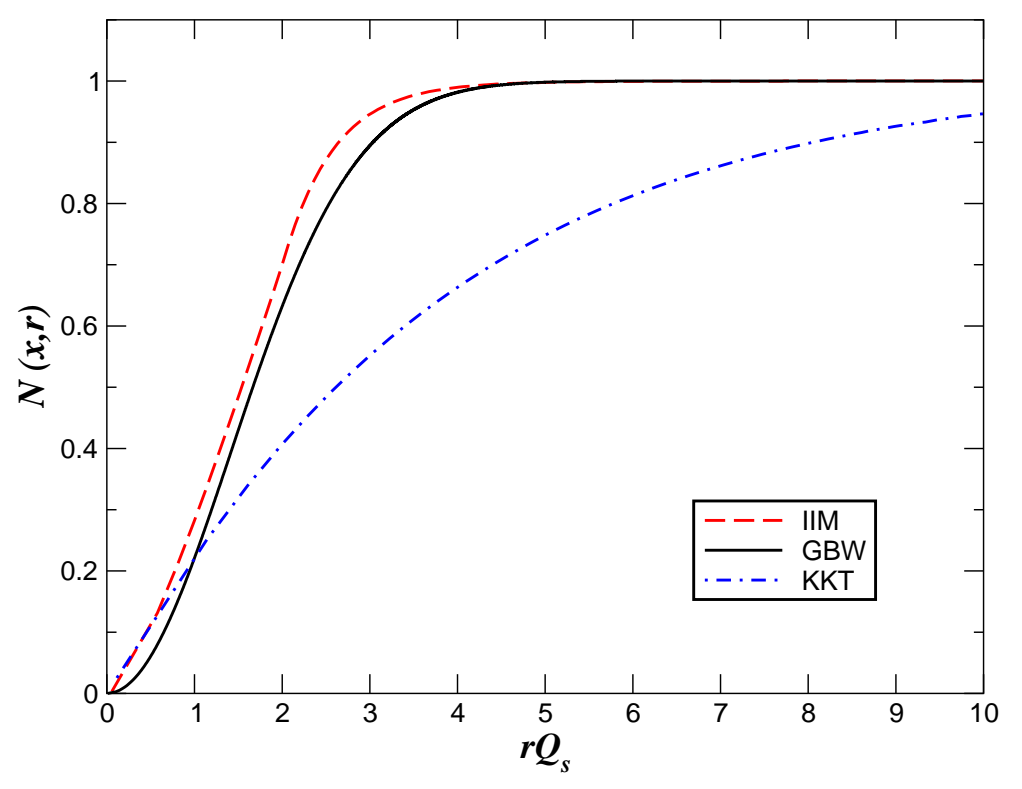

FIG. 3: Quark dipole scattering amplitude as a function of the scaling variable $\boldsymbol{r} Q_{s}$.

In Fig 2 we analyze the pair separation dependence of the quark dipole scattering amplitude for different values of $x$. As expected from the previous discussion, we observe that while the GBW and IIM parameterizations present a similar behavior for small $\boldsymbol{r}^{2}$, the KKT one predicts a smoother dependence. In the other limit, the GBW and IIM parameterizations saturate for large pair separations, while the KKT one still presents a residual dependence, demonstrating that the asymptotic regime is only reached for very large pair separations. The characteristic feature which is evident in the GBW and IIM models is that the dipole cross section saturates for smaller dipoles when $x$ assumes smaller values. An important aspect to be emphasized is the large difference between the predictions in the transition region, which we expect to be probed at HERA. For comparison we also present the predictions for $\mathcal{N}$ from the numerical solution of the BK equation as obtained in Ref. [38. We have that this solution has, as expected, the color transparency and saturation limits for small and large $\boldsymbol{r}$, respectively. However, the transition region is characterized by a sharp transition in the two values of $x$ shown. Moreover, it is important to emphasize the large difference between this results and the KKT prediction. In what follows we will restrict our analyzes for the phenomenological parameterizations which has its parameters fixed by the experimental data.

All models have the property of geometric scaling observed in the solutions of the BK equation. Mathematically, geometrical scaling means that the solution of the BK equation depends only on one combined variable $r Q_{s}(x)$ instead of $\boldsymbol{r}$ and $x$ separately, i. e., $\mathcal{N}(\boldsymbol{r}, x) \equiv \mathcal{N}\left(\boldsymbol{r} Q_{s}(x)\right)$. In Fig. 3 we show the dependence of the quark dipole scattering amplitude on the scaling variable $\boldsymbol{r} Q_{s}$. We observe that the three dipole scattering amplitudes grow in the region of small values of $\boldsymbol{r} Q_{s}$ as a power of $\boldsymbol{r} Q_{s}$, i.e. $\mathcal{N}(\boldsymbol{r}, x) \propto\left(\boldsymbol{r} Q_{s}\right)^{2 \gamma_{e f f}}$. However, $\gamma_{\text {eff }}$ is different in each model, being 1 for the GBW model, $\leq 1$ for the IIM model and about $\frac{1}{2}$ for the KKT one. This implies a different $\boldsymbol{r} Q_{s}$ dependence of the dipole scattering amplitudes and dipole cross sections. Since the saturation scale drives the energy dependence of the dipole cross section, these models present a very distinct energy dependence. This can clearly be seen in Fig. 4 where we present the $x$ dependence of the dipole scattering amplitudes for different values of the squared pair separation given by $\boldsymbol{r}^{2}=1 / Q^{2}$. We observe that for large $Q^{2}$ (small pair separation) the dipole scattering amplitude is dominated by the linear limit. Since the models have different behavior in this limit, the energy dependence is also different, with the GBW model presenting the strongest growth at small $x$. The behavior predicted by the IIM model is similar to the GBW one. On the other hand, the KKT model predicts the smallest growth with the energy. At large pair separations $\boldsymbol{r}>\boldsymbol{r}_{s}$, which characterizes the saturation regime, the GBW and IIM models predict the saturation of the dipole scattering ampitude, while the KKT one still presents a growth at small values $x$. Basically, the asymptotic saturation regime is only observed for very small values of $x$, beyond the kinematical range of HERA.

The basic observable measured with a great accuracy by HERA is the proton structure function $F_{2}\left(x, Q^{2}\right)$ which is directly related with the $\gamma^{*} p$ cross section by the following expression

$$
F_{2}\left(x, Q^{2}\right)=\frac{Q^{2}}{4 \pi^{2} \alpha_{e m}}\left(\sigma_{T}^{\gamma^{*} p}+\sigma_{L}^{\gamma^{*} p}\right) .
$$



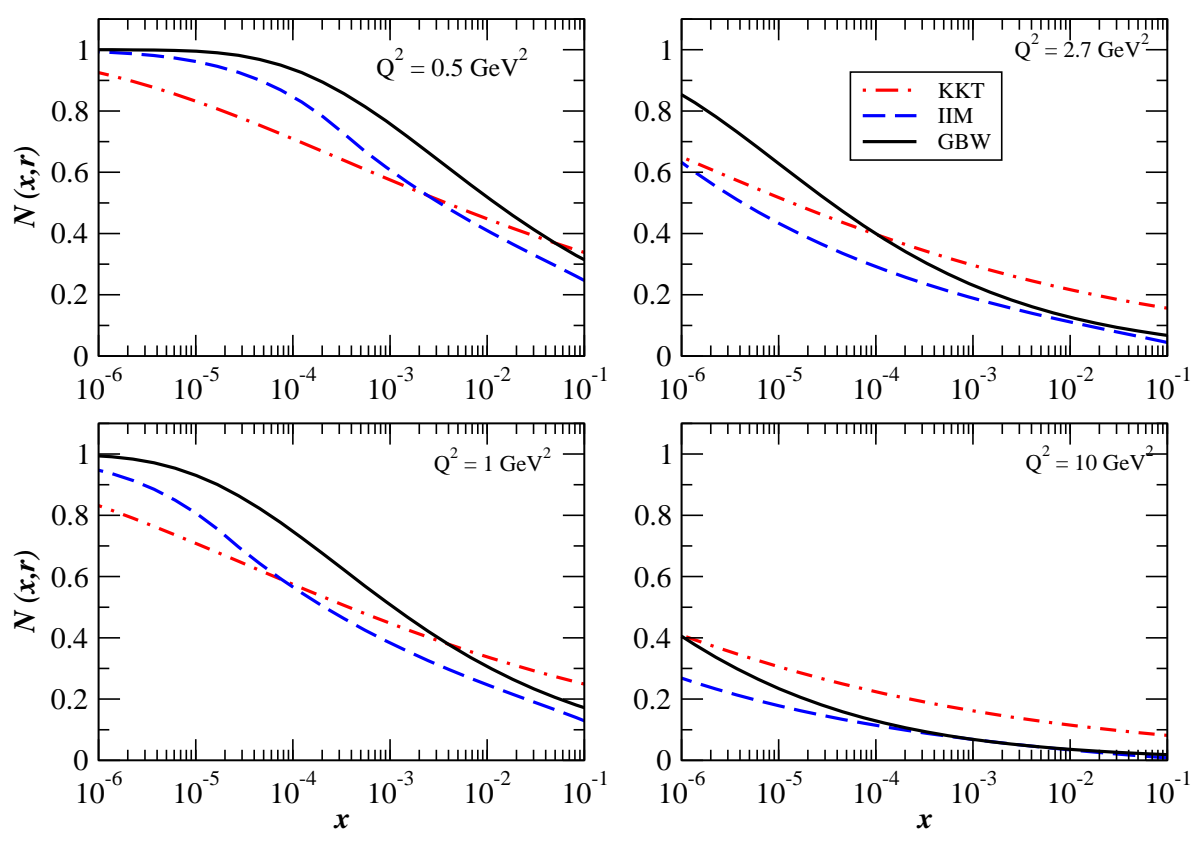

FIG. 4: Energy dependence of the quark dipole scattering amplitude for different values of the squared pair separation $\boldsymbol{r}^{2}=$ $1 / Q^{2}$.

Consequently, using the color dipole picture of DIS we can directly calculate $F_{2}$ for the different parameterizations of the quark dipole scattering ampitudes. Similarly, we can estimate the longitudinal structure function which is defined by $F_{L}\left(x, Q^{2}\right)=Q^{2} /\left(4 \pi^{2} \alpha_{e m}\right) \times \sigma_{L}^{\gamma^{*} p}$. In Figs. 5 and $[$ we present a comparison between the predictions of the different models and ZEUS data [46]. We have used that $\sigma_{d i p}(x, \boldsymbol{r})=\sigma_{0} \mathcal{N}(x, \boldsymbol{r})$ with $\sigma_{0}$ as given in the GBW and IIM parameterizations. For the KKT parameterization we have treated $\sigma_{0}$ as a free parameter and fixed its value by fitting the $F_{2}$ data at $Q^{2}=2.7 \mathrm{GeV}^{2}$. Our choice for this value of virtuality is justified by the fact that in this region we expect that the saturation physics should be dominant. We have tested other choices and verified that our main conclusion is not modified (see below). The predicitions for other values of virtualities are parameter free. Moreover, we have considered two different choices for the typical scale present in the process, needed to calculate the function $\gamma\left(Y, \boldsymbol{r}^{2}\right)$ in the KKT parameterization. Basically, we have assumed that $\boldsymbol{r} \approx 1 / Q_{s}$ or $\boldsymbol{r} \approx 1 / Q$. As we will demonstrate below, our predictions for $F_{2}$ in the kinematical range of interest are almost identical. We consider only few values of the photon virtuality in the region of low $Q^{2}$, where the saturation effects must be important. As expected, the GBW and IIM models describe quite well the experimental $F_{2}$ data (See Fig. 5) . On the other hand, the KKT parameterization is able to describe the experimental ep data only in a limited kinematical range of photon virtualities around of the virtuality where the normalization is fixed. The basic aspect of this parameterization is that the $Q^{2}$ dependence of the proton structure function cannot be described. Furthermore, this parameterization predicts an energy dependence, which is smoother than observed in the data. This behavior is directly related to the behavior present in the dipole scattering amplitude. The curve denoted KKTq in the figure represents the results obtained assuming $\boldsymbol{r} \approx 1 / Q$, while in the KKT curve we assume $\boldsymbol{r} \approx 1 / Q_{s}$ in the calculation of $\gamma\left(Y, \boldsymbol{r}^{2}\right)$. These two prescriptions differ appreciably only in the large $x$ and/or $Q^{2}$ region.

Finally, in Fig. [6] we present the predictions of the different models for the longitudinal structure function. For comparison, we also present the prediction obtained using the Altarelli-Martinelli equation and the GRV98 parameterization for the solution of the DGLAP evolution equation (for details see Ref. [45]). In this case we have that the KKT parameterization describe reasonably the few data available [47], similarly to the GBW and IIM parameterizations. However, this fact is mainly associated to the large experimental error in the current data. We believe that a future experimental study of the longitudinal structure function will be able to discriminate the parameterizations. 

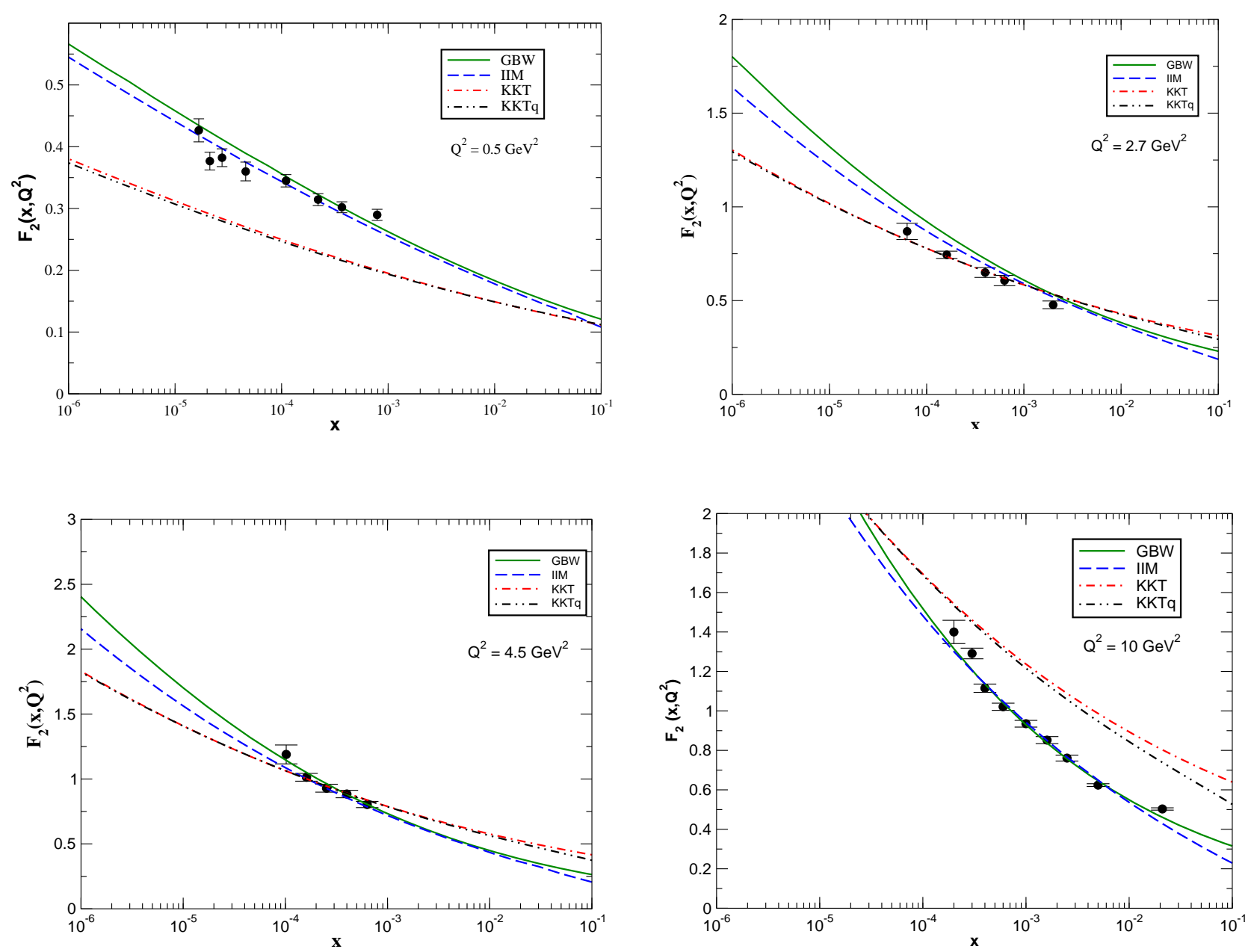

FIG. 5: Comparison between the predictions for $F_{2}\left(x, Q^{2}\right)$ of the distinct models at different values of $Q^{2}$. Data are from ZEUS.

\section{SUMMARY}

Assuming the universality of the hadron wavefunction predicted by the Color Glass Condensate formalism we can cross relate different experiments and gain a clear understading of the QCD dynamics at high energies. In this paper we have studied in detail different parameterizations of the dipole scattering ampitude proposed to describe the HERA and RHIC data. We have observed that these parameterizations predict distinct energy and dipole size dependences, mainly in the interpolation region between the linear and saturation regimes. Since the experimental data at HERA probe exactly this kinematical domain, a comparison with the $F_{2}$ data in the region of small $x$ and low $Q^{2}$ is very important, since it allows to discriminate between the parameterizations. We have concluded that the KKT parameterization is able to describe the experimental $e p$ data only in a limited kinematical range of photon virtualities. Therefore, the scaling violations of the proton structure function, observed in the HERA data, are not reproduced by this model. Moreover, the KKT parameterization predicts a smoother energy dependence than that observed in the data. As the IIM parameterization is not able to describe the RHIC data in the full kinematical range, our result put in check the property of universality, present in the CGC physics, for the current kinematical range of the RHIC and HERA experiments. In principle, it indicate that pre-asymptotic effects as for instance those associated to the different initial conditions present in $e p$ and $p A$ collisions, cannot still be disregarded and the cross relation between different experiments should be made with some caution as well as the interpretation of the comparison between the CGC predictions and the experimental data. We believe that an unified global fit of the RHIC and HERA data could be useful to obtain reliable predictions for the future colliders. 

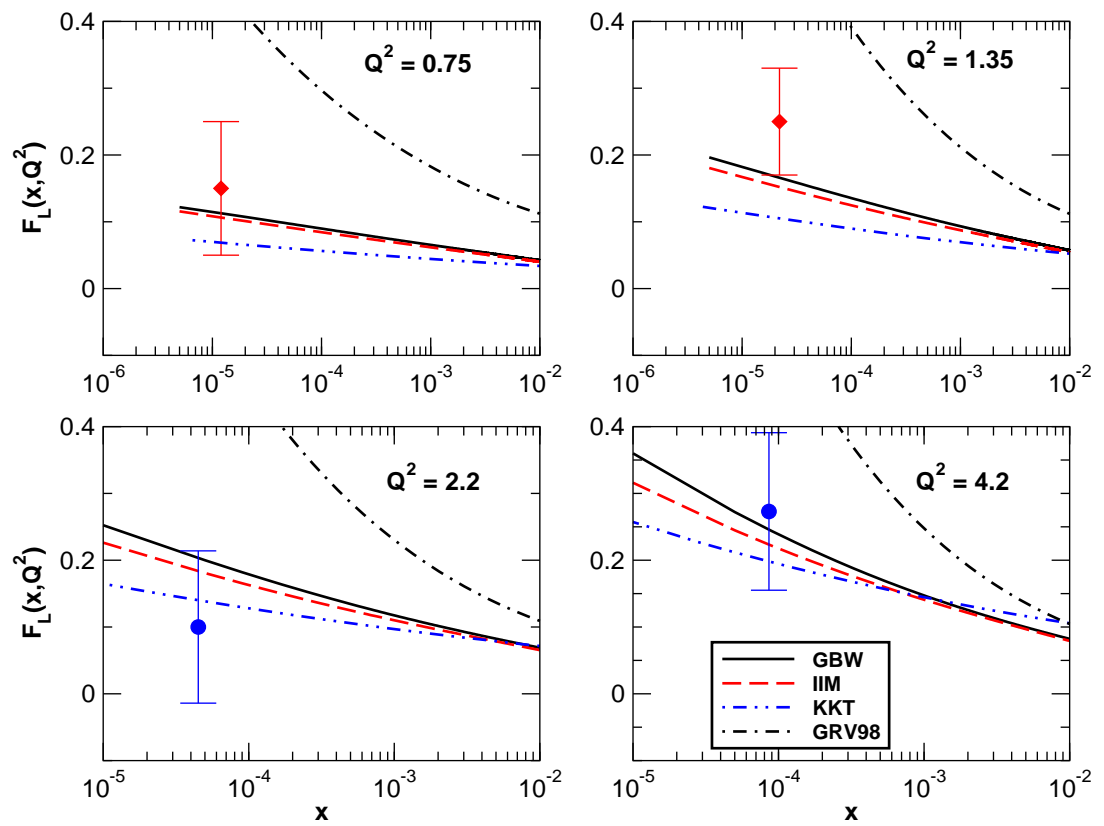

FIG. 6: Comparison between the predictions for $F_{L}\left(x, Q^{2}\right)$ of the distinct models at different values of $Q^{2}$.

\section{Acknowledgments}

The authors thank M. V. T. Machado and M. A. Betemps for helpful discussions. This work was partially financed by the Brazilian funding agencies CNPq, FAPESP and FAPERGS.

[1] V.N. Gribov and L.N. Lipatov, Sov. J. Nucl. Phys. 15, 438 (1972); G. Altarelli and G. Parisi, Nucl. Phys. B126, 298 (1977); Yu.L. Dokshitzer, Sov. Phys. JETP 46, 641 (1977).

[2] L. N. Lipatov, Sov. J. Nucl. Phys. 23, 338 (1976); E. A. Kuraev, L. N. Lipatov, V. S. Fadin, JETP 45, 1999 (1977); I. I. Balitskii, L. N. Lipatov, Sov. J. Nucl. Phys. 28, 822 (1978).

[3] M. Froissart, Phys. Rev. 123, 1053 (1961); A. Martin, Phys. Rev. 129, 1432 (1963).

[4] L.V. Gribov, E.M. Levin and M.G. Ryskin, Phys. Rep. 100, 1 (1983).

[5] A.H. Mueller and Jian-wei Qiu, Nucl. Phys. B268, 427 (1986).

[6] J. Bartels, Z. Phys. C 60, 471 (1993); ibid. 62, 425 (1994); J. Bartels and M. Wüsthoff, Z. Phys. C 66, 157 (1995); J. Bartels and C. Ewerz, JHEP 9909, 026 (1999).

[7] A. L. Ayala Filho, M. B. Gay Ducati and E. M. Levin, Nucl. Phys. B493, 305 (1997), ibid. B511, 355 (1998).

[8] L. McLerran and R. Venugopalan, Phys. Rev. D 49, 2233 (1994), ibid. 49, 3352 (1994), ibid. 50, 2225 (1994).

[9] I. I. Balitsky, Nucl. Phys. B463, 99 (1996), Phys. Rev. Lett. 81, 2024 (1998), Phys. Rev. D 60, 014020 (1999), Phys. Lett. B 518, 235 (2001); I.I. Balitsky and A.V. Belitsky, Nucl. Phys. B629, 290 (2002).

[10] E. Iancu, A. Leonidov and L. McLerran, Nucl.Phys. A692 (2001) 583; E. Ferreiro, E. Iancu, A. Leonidov and L. McLerran, Nucl. Phys. A701, 489 (2002).

[11] J. Jalilian-Marian, A. Kovner, L. McLerran and H. Weigert, Phys. Rev. D 55, 5414 (1997); J. Jalilian-Marian, A. Kovner and H. Weigert, Phys. Rev. D 59, 014014 (1999), ibid. 59, 014015 (1999), ibid. 59034007 (1999); A. Kovner, J. Guilherme Milhano and H. Weigert, Phys. Rev. D 62, 114005 (2000); H. Weigert, Nucl. Phys. A703, 823 (2002).

[12] Y.V. Kovchegov, Phys. Rev. D 60, 034008 (1999), ibid. 61074018 (2000).

[13] M. A. Braun, Eur. Phys. J. C 16, 337 (2000); N. Armesto and M. A. Braun, Eur. Phys. J. C 20, 517 (2001).

[14] E. Iancu and R. Venugopalan, arXiv:hep-ph/0303204

[15] A. M. Stasto, Acta Phys. Polon. B 35, 3069 (2004).

[16] H. Weigert, arXiv:hep-ph/0501087

[17] J. Jalilian-Marian and Y. V. Kovchegov, arXiv:hep-ph/0505052

[18] E. Iancu, K. Itakura, S. Munier, Phys. Lett. B590,199 (2004).

[19] N. Armesto, C. A. Salgado and U. A. Wiedemann, Phys. Rev. Lett. 94, 022002 (2005)

[20] E. Iancu, K. Itakura and L. McLerran, Nucl. Phys A708, 327 (2002). 
[21] J. Kwiecinski and A. M. Stasto, Phys. Rev. D 66, 014013 (2002).

[22] V. P. Goncalves and M. V. T. Machado, Mod. Phys. Lett. 19, 2525 (2004)

[23] K. Golec-Biernat and M. Wüsthoff, Phys. Rev. D 59, 014017 (1999), ibid. D60 114023 (1999).

[24] J. Bartels, K. Golec-Biernat, H. Kowalski, Phys. Rev. D 66, 014001 (2002).

[25] H. Kowalski and D. Teaney, Phys. Rev. D 68, 114005 (2003)

[26] J. R. Forshaw and G. Shaw, JHEP 0412, 052 (2004)

[27] A. M. Staśto, K. Golec-Biernat and J. Kwieciński, Phys. Rev. Lett. 86, 596 (2001).

[28] V. P. Goncalves and M. V. T. Machado, Phys. Rev. Lett. 91, 202002 (2003)

[29] I. Arsene et al. [BRAHMS Collaboration], Phys. Rev. Lett. 91, 072305 (2003); Phys. Rev. Lett. 93, 242303 (2004); Phys. Rev. Lett. 94, 032301 (2005); arXiv:nucl-ex/0410020

[30] R. Baier, A. Kovner and U. A. Wiedemann, Phys. Rev. D 68, 054009 (2003); J. Jalilian-Marian, Y. Nara and R. Venugopalan, Phys. Lett. B 577, 54 (2003); D. Kharzeev, Y. V. Kovchegov and K. Tuchin, Phys. Rev. D 68, 094013 (2003); J. L. Albacete, N. Armesto, A. Kovner, C. A. Salgado and U. A. Wiedemann, Phys. Rev. Lett. 92, 082001 (2004); E. Iancu, K. Itakura and D. N. Triantafyllopoulos, Nucl. Phys. A 742, 182 (2004).

[31] J. Jalilian-Marian, Nucl. Phys. A 748, 664 (2005)

[32] D. Kharzeev, Y.V. Kovchegov and K. Tuchin, Phys. Lett. B599, 23 (2004).

[33] A. Dumitru, A. Hayashigaki and J. Jalilian-Marian, arXiv:hep-ph/0506308

[34] J. Jalilian-Marian, private communication.

[35] M. A. Betemps and M. B. Gay Ducati, Phys. Rev. D 70, 116005 (2004)

[36] J. Jalilian-Marian, Nucl. Phys. A 753, 307 (2005)

[37] M. A. Kimber, J. Kwiecinski and A. D. Martin, Phys. Lett. B 508, 58 (2001); E. Levin and M. Lublinsky, Nucl. Phys. A 696, 833 (2001); M. Lublinsky, Eur. Phys. J. C 21, 513 (2001); M. Lublinsky, E. Gotsman, E. Levin and U. Maor, Nucl. Phys. A 696, 851 (2001); K. Golec-Biernat, L. Motyka and A. M. Stasto, Phys. Rev. D 65, 074037 (2002); K. GolecBiernat and A. M. Stasto, Nucl. Phys. B 668, 345 (2003); K. Rummukainen and H. Weigert, Nucl. Phys. A 739, 183 (2004); E. Gotsman, M. Kozlov, E. Levin, U. Maor and E. Naftali, Nucl. Phys. A 742, 55 (2004); J. L. Albacete, N. Armesto, J. G. Milhano, C. A. Salgado and U. A. Wiedemann, Phys. Rev. D 71, 014003 (2005) K. Kutak and A. M. Stasto, Eur. Phys. J. C 41, 343 (2005); G. Chachamis, M. Lublinsky and A. Sabio Vera, Nucl. Phys. A 748, 649 (2005); T. Ikeda and L. McLerran, Nucl. Phys. A 756, 385 (2005); C. Marquet and G. Soyez, arXiv:hep-ph/0504080 R. Enberg, K. GolecBiernat and S. Munier, arXiv:hep-ph/0505101

[38] E. Gotsman, E. Levin, M. Lublinsky and U. Maor, Eur. Phys. J. C 27, 411 (2003)

[39] N. N. Nikolaev and B. G. Zakharov, Z. Phys. C49, 607 (1991); Z. Phys. C53, 331 (1992); A. H. Mueller, Nucl. Phys. B415, 373 (1994); A. H. Mueller and B. Patel, Nucl. Phys. B425, 471 (1994).

[40] V. Barone and E. Predazzi, High-Energy Particle Diffraction, Springer-Verlag, Berlin Heidelberg, (2002).

[41] A.H. Mueller and D.N. Triantafyllopoulos, Nucl. Phys. B640, 331 (2002); D.N. Triantafyllopoulos, Nucl. Phys. B648, 293 (2003); A.H. Mueller, Nucl. Phys. A724, 223 (2003).

[42] E. Levin and K. Tuchin, Nucl. Phys. B 573, 833 (2000).

[43] J. R. Forshaw, R. Sandapen and G. Shaw, Phys. Rev. D 69, 094013 (2004).

[44] J. R. Forshaw, R. Sandapen and G. Shaw, Phys. Lett. B 594, 283 (2004).

[45] V. P. Goncalves and M. V. T. Machado, Eur. Phys. J. C 37, 299 (2004)

[46] S. Chekanov et al. [ZEUS Collaboration], Eur. Phys. J. C 21, 443 (2001)

[47] C. Adloff et al. [H1 Collaboration], Eur. Phys. J. C 21, 33 (2001); E. M. Lobodzinska, Acta Phys. Polon. B 35 (2004) 223 ; arXiv:hep-ph/0311180 